\title{
Comparison of Adomian decomposition and Homotopy perturbation methods for higher-order linear fractional integro- differential equations
}

\author{
M.H. Saleh ${ }^{1}$, S.M. Amer ${ }^{1}$ and M.A. Shaalan ${ }^{2, *}$ \\ ${ }^{l}$ (Mathematics Department, Faculty of Science, Zagazig University, Zagazig, Egypt) \\ ${ }^{2}$ (Basic Science Department, Higher Technological Institute, Tenth of Ramadan City, Egypt)
}

\begin{abstract}
In this paper, we will compare between Adomian decomposition method (ADM) and Homotopy perturbation method (HPM) for obtaining the numerical solutions of higher-order linear fractional integro-differential equations with boundary conditions. Numerical examples are presented to illustrate the efficiency and accuracy of the proposed methods.
\end{abstract}

Keywords: Adomian decomposition method, Homotopy perturbation method, Boundary value problems, Fractional integro-differential equations, Caputo fractional derivative.

\section{Introduction}

Fractional differential equations have attracted much attention which provides an efficient for the description of many practical dynamical phenomena arising in engineering and scientific disciplines such as, physics, biology, chemistry, economy, electrochemistry, electromagnetic, control theory, viscoelasticity, see [16]. Many mathematical formulations of physical phenomena lead to integro-differential equations such as, fluid dynamics, continuum and statistical mechanics, see [7-11].

In this paper, we considered the linear boundary value problems for higher-order fractional integro-differential equations with a Caputo fractional derivative of the type:

$$
D^{\alpha} y(x)=f(x)+\gamma y(x)+\lambda \int_{0}^{x} k(x, t) y(t) d t, 0<x<b, m-1<\alpha \leq m, m \in Z^{+},
$$

subject to the following boundary conditions:

$$
\begin{array}{ll}
y(0)=\gamma_{0}, & y^{(i)}(0)=\gamma_{i}, \\
y(b)=\beta_{0}, & y^{(i)}(b)=\beta_{i},
\end{array}
$$

where $D^{\alpha}$ is a caputo fractional derivative, $\gamma_{0}, \gamma_{i}, \beta_{0}, \beta_{i}, \lambda$ and $\gamma$ are real constants $\left(i=2 k, k \in Z^{+}, 1 \leq \mathrm{k}<\right.$ $m / 2), f(x)$ and $k(x, t)$ are given and can be approximated by Taylor polynomials. The existence and stability of solutions for fractional integro-differential equations [12-14]. He [15-19] was the first to propose the Adomian decomposition method (ADM) and homotopy perturbation method (HPM) for finding the solutions of non-linear problems. Most fractional integro-differential equations do not have exact solutions, so must we use the approximate techniques. There are many methods for seeking approximate solutions such as variational iteration method, homotopy perturbation method, homotopy analysis method, the fractional differential transform method and Adomian decomposition method, see[20- 23].The outline of this paper is as follows: In section 2,we present some preliminaries. Section 3, contains the application of the Adomian decomposition method. Section 4, contains the application of the homotopy perturbation method. Finally, Section 5, devoted to illustrate some numerical examples on mentioned methods.

\section{Preliminaries}

Definition 2.1. A real function $f(x), x>0$, is said to be in the space $C_{\alpha}, \alpha \in R$, if there exists a real number $p>\alpha$, such that $f(x)=x^{p} f_{1}(x)$, where $f_{1}(x) \in C[0, \infty)$.

Definition 2.2. A real function $f(x), x>0$, is said to be in the space $C_{\alpha}^{k}, k \in N$, if $f^{k} \in C_{\alpha}$.

Definition 2.3. $I^{\alpha}$ denotes the fractional integral operator of order $\alpha$ in the sense of Riemann-Liouville, defined by: 
$I^{\alpha} f(x)= \begin{cases}\frac{1}{\Gamma(\alpha)} \int_{0}^{x} \frac{f(t)}{(x-t)^{1-\alpha}} d t, & \alpha>0, \\ f(x), & \alpha=0 .\end{cases}$

Definition 2.4. Let $f \in C_{-1}^{m}, \mathrm{~m} \in N$. Then the Caputo fractional derivative of $f(x)$, defined by:

$D^{\alpha} f(x)=\left\{\begin{array}{lc}\frac{1}{\Gamma(m-\alpha)} \int_{0}^{x} \frac{f^{(m)}(t)}{(x-t)^{\alpha-m+1}} d t, & 0 \leq m-1<\alpha \leq m, \\ \frac{d^{m} f(x)}{d x^{m}}, & \alpha=m \in N .\end{array}\right.$

Now, we introduce some basic properties of fractional operator are listed below [1]:

For $f \in C_{\mu}, \mu \geq-1, \gamma \geq-1, \alpha, \beta \geq 0$ :

(1) $I^{\alpha} I^{\beta} f(x)=I^{\alpha+\beta} f(x)=I^{\beta} I^{\alpha} f(x)$.

(2) $I^{\alpha} x^{\gamma}=\frac{\Gamma(\gamma+1)}{\Gamma(\alpha+\gamma+1)} x^{\alpha+\gamma}$.

(3) $D^{\alpha}\left[I^{\alpha} f(x)\right]=f(x)$.

(4) $I^{\delta}\left[D^{\alpha} f(x)\right]=f(x)-\sum_{k=0}^{m-1} f^{k}(0) \frac{x^{k}}{k !}, 0 \leq m-1<\alpha \leq m \in N$.

\section{Adomian decomposition method}

Consider the equation (1) with boundary conditions (2), (3). Applying the integral operator $I^{\alpha}$ to both sides of the equation (1), we get:

$$
y(x)=\sum_{j=0}^{i} \gamma_{j} \frac{x^{j}}{j !}+I^{\alpha}[f(x)+\gamma y(x)]+I^{\alpha}\left[\lambda \int_{0}^{x} k(x, t) y(t) d t\right],
$$

according to the Adomian decomposition method $[15,16]$, we put the solution $y(x)$ be decomposed by infinite series of components as follows:

$y(x)=\sum_{n=0}^{\infty} y_{n}(x)$.

Substitution the decomposition (8) into both sides of (7), we get:

$$
\sum_{n=0}^{\infty} y_{n}(x)=\sum_{j=0}^{i} \gamma_{j} \frac{x^{j}}{j !}+I^{\alpha}\left[f(x)+\gamma \sum_{n=0}^{\infty} y_{n}(x)\right]+I^{\alpha}\left[\lambda \int_{0}^{x} k(x, t) \sum_{n=0}^{\infty} y_{n}(t) d t\right] \text {. }
$$

From equation (9), the iterations are determined as follows:

$$
\begin{aligned}
& y_{0}(x)=\sum_{j=0}^{i} \gamma_{j} \frac{x^{j}}{j !}+I^{\alpha}[f(x)], \\
& y_{n+1}(x)=\gamma I^{\alpha}\left[y_{n}(x)\right]+I^{\alpha}\left[\lambda \int_{0}^{x} k(x, t) y_{n}(t) d t\right], n \geq 0 .
\end{aligned}
$$

Where $\gamma_{1}=y^{\prime}(0), \gamma_{3}=y^{\prime \prime \prime}(0), \cdots$ and $\gamma_{i}=y^{(i)}(0)$ are to be determined. The decomposition series solutions are generally converging very rapidly [24-29], we approximate the series solution of ADM by the following $N$-terms truncated series:

$\Phi_{N}(x)=y_{0}(x)+y_{1}(x)+y_{2}(x)+\cdots+y_{N-1}(x)$.

Substitution (3) in (12), we get the following system of equations:

$$
\begin{aligned}
& y_{0}(b)+y_{1}(b)+y_{2}(b)+\cdots+y_{N-1}(b)=\beta_{0}, \\
& y_{0}^{\prime \prime}(b)+y_{1}^{\prime \prime}(b)+y_{2}^{\prime \prime}(b)+\cdots+y_{N-1}^{\prime \prime}(b)=\beta_{2}, \\
& \vdots \\
& y^{(i)}{ }_{0}(b)+y^{(i)}{ }_{1}(b)+y^{(i)}{ }_{2}(b)+\cdots+y^{(i)}{ }_{N-1}(b)=\beta_{i} .
\end{aligned}
$$


From the system of equations (13), we can find the unknowns $\gamma_{1}, \gamma_{3}, \ldots, \gamma_{i}$. Substitution the constant values of $\gamma_{1}, \gamma_{3}, \ldots, \gamma_{i}$ in equation (12), we get the approximate solution of the problem (1) - (3).

\section{Homotopy perturbation method}

Consider the equation (1) with boundary value conditions (2), (3). According to HPM [17 - 19], we construct the following homotopy:

$(1-P) \mathrm{D}^{\alpha} y(x)+P\left(\mathrm{D}^{\alpha} y(x)-f(x)-\gamma y(x)-\lambda \int_{0}^{x} k(x, t) y(t) d t\right)=0$,

or

$\mathrm{D}^{\alpha} y(x)=P\left(f(x)+\gamma y(x)+\lambda \int_{0}^{x} k(x, t) y(t) d t\right)$,

where $P \in[0,1]$ is an embedding parameter. If $P=0$, then equation (15) becomes a linear equation,

$D^{\alpha} y(x)=0$,

and when $P=1$, then the equation (15) becomes the original equation (1). The solution of equation (1) can be written as a power series in $P$ as follows:

$y(x)=y_{0}(x)+P y_{1}(x)+P^{2} y_{2}(x)+P^{3} y_{3}(x)+\cdots \cdots$

Put $P=1$ in equation (17), so the approximate solution of equation (1) is:

$y(x)=y_{0}(x)+y_{1}(x)+y_{2}(x)+y_{3}(x)+\cdots \cdots$

The convergence of the series (18) is proven in [30]. Substituting (17) in (15), then equating the terms with identical power of $P$, we obtain the series of linear equations:

$P^{0}: D^{\alpha} y_{0}(x)=0$,

$P^{1}: \mathrm{D}^{\alpha} y_{1}(x)=f(x)+\gamma y_{0}(x)+\lambda \int_{0}^{x} k(x, t) y_{0}(t) d t$,

$P^{2}: \mathrm{D}^{\alpha} y_{2}(x)=\gamma y_{1}(x)+\lambda \int_{0}^{x} k(x, t) y_{1}(t) d t$,

$P^{3}: \mathrm{D}^{\alpha} y_{3}(x)=\gamma y_{2}(x)+\lambda \int_{0}^{x} k(x, t) y_{2}(t) d t$, $\vdots$

From equation (19), the initial approximation can be chosen as follows:

$y_{0}(x)=\sum_{j=0}^{i} \gamma_{j} \frac{x^{j}}{j !}, \quad\left(i=2 k, k \in Z^{+}, 1 \leq \mathrm{k}<m / 2\right)$

where $\gamma_{1}=y^{\prime}(0), \gamma_{3}=y^{\prime \prime \prime}(0), \cdots$ and $\gamma_{i}=y^{(i)}(0)$ are to be determined by applying boundary conditions (3).

Equation (19) and system of equations (20) can be solved by applying the integral operator $I^{\alpha}$, and then by using simple computation, we approximate the series solution of HPM by the following $N-$ terms truncated series:

$\Phi_{N}(x)=y_{0}(x)+y_{1}(x)+y_{2}(x)+\cdots+y_{N-1}(x)$.

Substitution (3) in (22), we get the following system of equations:

$$
\begin{aligned}
& y_{0}(b)+y_{1}(b)+y_{2}(b)+\cdots+y_{N-1}(b)=\beta_{0}, \\
& y_{0}^{\prime \prime}(b)+y_{1}^{\prime \prime}(b)+y_{2}^{\prime \prime}(b)+\cdots+y_{N-1}^{\prime \prime}(b)=\beta_{2}, \\
& \vdots \\
& y^{(i)}{ }_{0}(b)+y^{(i)}{ }_{1}(b)+y^{(i)}(b)+\cdots+y^{(i)}{ }_{N-1}(b)=\beta_{i} .
\end{aligned}
$$

From the system of equations (23), we can find the unknowns $\gamma_{1}, \gamma_{3}, \ldots, \gamma_{i}$. Substitution the constant values of $\gamma_{1}, \gamma_{3}, \ldots, \gamma_{i}$ in equation (22), we get the approximate solution of the problem (1)-(3). 


\section{Numerical examples}

In this section we will apply ADM and HPM for higher-order fractional integro-differential equations with known exact solutions at $\alpha=4, \alpha=6$. All results are obtained by using Maple 16:

Example1. Consider the following linear fourth-order fractional integro-differential equation:

$$
D^{\alpha} y(x)=1-(1+x) e^{-x}-\int_{0}^{x} e^{-t} y(t) d t, 0<x<1,3<\alpha \leq 4,
$$

subject to the following boundary conditions:

$$
\begin{array}{ll}
y(0)=0, & \mathrm{y}^{\prime \prime}(0)=0 \\
y(1)=1, & \mathrm{y}^{\prime \prime}(1)=0 .
\end{array}
$$

For $\alpha=4$, the exact solution of the above problem (24) - (26) is $y(x)=x$.

According to ADM, the recursive Adomian decomposition algorithm is:

$$
\begin{aligned}
& y_{0}(x)=\sum_{j=0}^{3} \gamma_{j} \frac{x^{j}}{j !}+I^{\alpha}[f(x)], \\
& \vdots \\
& y_{n+1}(x)=I^{\alpha}\left[-\int_{0}^{x} e^{-t} y_{n}(t) d t\right] .
\end{aligned}
$$

Where $A=y^{\prime}(0), B=y^{\prime \prime \prime}(0)$ is to be determined. To avoid difficult fractional integration, we can take the truncated Taylor expansions for the exponential term: e.g. $e^{ \pm x}=1 \pm x+x^{2} / 2 ! \pm x^{3} / 3$ !. Thus, by solving (27), (28), we can form the $N$-terms approximation, $N=2$ :

$$
\begin{aligned}
& y_{0}(x)=A x+\frac{B x^{3}}{6}+\frac{x^{\alpha+2}}{\Gamma(\alpha+3)}-\frac{2 x^{\alpha+3}}{\Gamma(\alpha+4)}+\frac{4 x^{\alpha+4}}{\Gamma(\alpha+5)}, \\
& \Phi_{2}(x)=y_{0}(x)+I^{\alpha}\left[-\int_{0}^{x} e^{-t} y_{0}(t) d t\right] .
\end{aligned}
$$

Where $A, \mathrm{~B}$ can be determined by using boundary condition (26) in $\Phi_{2}(x)$ (see Table 1).

Table1. Values of $A$ and $B$ for different values of $\alpha$ using (29).

\begin{tabular}{ccccc}
\hline & $\alpha=3.25$ & $\alpha=3.50$ & $\alpha=3.75$ & $\alpha=4$ \\
\hline$A$ & 1.001289323 & 1.000824834 & 1.000521904 & 1.000326805 \\
$B$ & -0.008770162045 & -0.005565630077 & -0.003495472122 & -0.002173843410 \\
\hline
\end{tabular}

We compute the absolute error functions $E_{1}(x)=\left|x-\Phi_{2,3.25}\right|, E_{2}(x)=\left|x-\Phi_{2,3.50}\right|$ and $E_{3}(x)=\left|x-\Phi_{2,3.75}\right|$. Where $x$ is the exact solution of (24)-(26) and $\Phi_{2,3.25}, \Phi_{2,3.50}$ and $\Phi_{2,3.75}$ are approximate solutions of (24) (26) by using (29) at $\alpha=3.25, \alpha=3.50$ and $\alpha=3.75$ respectively.

According to HPM, we construct the following homotopy:

$$
D^{\alpha} y(x)=P\left(1-x e^{-x}-e^{-x}-\int_{0}^{x} e^{-t} y(t) d t\right) \text {. }
$$

Substituting (17) in (30), we obtain the following series of linear equations with identical power of $P$ :

$$
\begin{aligned}
& P^{0}: D^{\alpha} y_{0}(x)=0, \\
& P^{1}: \mathrm{D}^{\alpha} y_{1}(x)=1-x e^{-x}-e^{-x}-\int_{0}^{x} e^{-t} y_{0}(t) d t, \\
& P^{2}: \mathrm{D}^{\alpha} y_{2}(x)=-\int_{0}^{x} e^{-t} y_{1}(t) d t,
\end{aligned}
$$$$
\}
$$

Applying the operator $I^{\alpha}$ to the above series of linear equations and using the initial condition (25), we get:

$$
y_{0}(x)=0 \text {, }
$$




$$
\begin{aligned}
& y_{1}(x)=A x+\frac{B x^{3}}{6}+I^{\alpha}\left[1-x e^{-x}-e^{-x}-\int_{0}^{x} e^{-t} y_{0}(t) d t\right] \\
& y_{2}(x)=I^{\alpha}\left[-\int_{0}^{x} e^{-t} y_{1}(t) d t\right], \\
& \vdots
\end{aligned}
$$

Where $A=y^{\prime}(0), B=y^{\prime \prime \prime}(0)$ is to be determined. To avoid difficult fractional integration, we can take the truncated Taylor expansions for the exponential term in the system (34): e.g. $e^{ \pm x}=1 \pm x+x^{2} / 2$ ! $\pm x^{3} / 3$ !. Thus, by solving (33), (34), we obtain $y_{1}, y_{2}, \ldots$.

$$
y_{1}(x)=A x+\frac{B x^{3}}{6}+\frac{x^{\alpha+2}}{\Gamma(\alpha+3)}-\frac{2 x^{\alpha+3}}{\Gamma(\alpha+4)}+\frac{4 x^{\alpha+4}}{\Gamma(\alpha+5)},
$$

Now, we can form the $N$-terms approximation, $N=2$ :

$$
\Phi_{2}(x)=A x+\frac{B x^{3}}{6}+\frac{x^{\alpha+2}}{\Gamma(\alpha+3)}-\frac{2 x^{\alpha+3}}{\Gamma(\alpha+4)}+\frac{4 x^{\alpha+4}}{\Gamma(\alpha+5)} \text {. }
$$

Where $A$, B can be determined by using boundary condition (26) in $\Phi_{2}(x)$ (see Table 2).

Table2. Values of $A, B$ for different values of $\alpha$ using (36).

\begin{tabular}{ccccc}
\hline & $\alpha=3.25$ & $\alpha=3.50$ & $\alpha=3.75$ & $\alpha=4$ \\
\hline$A$ & 1.010100244 & 1.007586266 & 1.005567011 & 1.004001323 \\
$B$ & -0.08553725904 & -0.06165650476 & -0.04373516366 & -0.03055555556 \\
\hline
\end{tabular}

We compute the absolute error functions $E_{4}(x)=\left|x-\Phi_{2,3.25}\right|, E_{5}(x)=\left|x-\Phi_{2,3.50}\right|$ and $E_{6}(x)=\left|x-\Phi_{2,3.75}\right|$. Where $x$ is the exact solution of (24) - (26) and $\Phi_{2,3.25}, \Phi_{2,3.50}$ and $\Phi_{2,3.75}$ are approximate solutions of (24) (26) by using (36) at $\alpha=1.25, \alpha=1.50$ and $\alpha=1.75$ respectively. In Fig. 1 we compare the absolute error functions.

Example2. Consider the following linear sixth-order fractional integro-differential equation:

$D^{\alpha} y(x)=-1+(2-x) e^{x}+\int_{0}^{x} t y(t) d t, 0<x<1,5<\alpha \leq 6$,

subject to the following boundary conditions:

$$
\begin{array}{lll}
y(0)=1, & y^{\prime \prime}(0)=1, & y^{(4)}(0)=1 \\
y(1)=e, & y^{\prime \prime}(1)=e, & y^{(4)}(1)=e .
\end{array}
$$

For $\alpha=6$, the exact solution of the above problem (37) - (39) is $y(x)=e^{x}$.

According to ADM, the recursive Adomian decomposition algorithm is:

$$
\begin{aligned}
& y_{0}(x)=\sum_{j=0}^{5} \gamma_{j} \frac{x^{j}}{j !}+I^{\alpha}[f(x)], \\
& \vdots \\
& y_{n+1}(x)=I^{\alpha}\left[\int_{0}^{x} t y(t) d t\right] .
\end{aligned}
$$

Where $A=y^{\prime}(0), B=y^{\prime \prime \prime}(0)$ and $C=y^{(5)}(0)$ are to be determined. To avoid difficult fractional integration, we can take the truncated Taylor expansions for the exponential term: e.g. $e^{x}=1+x+x^{2} / 2 !+x^{3} / 3$ !. Thus, by solving (40), (41), we can form the $N$-terms approximation, $N=2$ :

$$
y_{0}(x)=1+A x+\frac{B x^{3}}{6}+\frac{x^{4}}{24}+\frac{C x^{5}}{120}+\frac{x^{\alpha}}{\Gamma(\alpha+1)}+\frac{x^{\alpha+1}}{\Gamma(\alpha+2)}-\frac{x^{\alpha+3}}{\Gamma(\alpha+4)}-\frac{4 x^{\alpha+4}}{\Gamma(\alpha+5)},
$$


$\Phi_{2}(x)=y_{0}(x)+I^{\alpha}\left[\int_{0}^{x} t y_{0}(t) d t\right]$.

Where $A, B$ and $C$ can be determined by using boundary condition (39) in $\Phi_{2}(x)$, (see Table 3).

Table3. Values of $A, B$ and $C$ for different values of $\alpha$ using (42).

\begin{tabular}{ccccc}
\hline & $\alpha=5.25$ & $\alpha=5.50$ & $\alpha=5.75$ & $\alpha=6$ \\
\hline$A$ & 0.9987943931 & 0.9985646077 & 0.9990890315 & 1.000033984 \\
$B$ & 1.013697556 & 1.015730408 & 1.009763351 & 0.9996955212 \\
$C$ & 0.2955891970 & 0.5610041471 & 0.7981238648 & 1.002067372 \\
\hline
\end{tabular}

We compute the absolute error functions $E_{7}(x)=\left|e^{x}-\Phi_{2,5.25}\right|, E_{8}(x)=\left|e^{x}-\Phi_{2,5.50}\right|$ and $E_{9}(x)=\left|e^{x}-\Phi_{2,5.75}\right|$. Where $e^{x}$ is the exact solution of (37) - (39) and $\Phi_{2,5.25}, \Phi_{2,5.50}$ and $\Phi_{2,5.75}$ are approximate solutions of (37) (39) by using (42) at $\alpha=3.25, \alpha=3.50$ and $\alpha=3.75$ respectively.

According to HPM, we construct the following homotopy:

$D^{\alpha} y(x)=P\left(-1+(2-x) e^{x}+\int_{0}^{x} t y(t) d t\right)$.

Substituting (17) in (43), we obtain the following series of linear equations with identical power of $P$ :

$P^{0}: D^{\alpha} y_{0}(x)=0$,

$P^{1}: \mathrm{D}^{\alpha} y_{1}(x)=-1+(2-x) e^{x}+\int_{0}^{x} t y_{0}(t) d t$,

$P^{2}: \mathrm{D}^{\alpha} y_{2}(x)=\int_{0}^{x} t y_{1}(t) d t$

Applying the operator $I^{\alpha}$ to the above series of linear equations and using the initial condition (38), we get:

$y_{0}(x)=1$,

$$
\left.\begin{array}{l}
y_{1}(x)=1+A x+\frac{B x^{3}}{6}+\frac{x^{4}}{24}+\frac{C x^{5}}{120}+I^{\alpha}\left[-1+(2-x) e^{x}+\int_{0}^{x} t y_{0}(t) d t\right], \\
y_{2}(x)=I^{\alpha}\left[\int_{0}^{x} t y_{1}(t) d t\right],
\end{array}\right\}
$$

Where $A=y^{\prime}(0), B=y^{\prime \prime \prime}(0)$ and $C=y^{(5)}(0)$ are to be determined. To avoid difficult fractional integration, we can take the truncated Taylor expansions for the exponential term in the system (47): e.g. $e^{x}=1+x+x^{2} / 2 !+x^{3} / 3$ !. Thus, by solving (46), (47), we obtain $y_{1}, y_{2}, \ldots$

$y_{1}(x)=A x+\frac{x^{2}}{2}+\frac{B x^{3}}{6}+\frac{x^{4}}{24}+\frac{C x^{5}}{120}+\frac{x^{\alpha}}{\Gamma(\alpha+1)}+\frac{x^{\alpha+1}}{\Gamma(\alpha+2)}+\frac{x^{\alpha+2}}{\Gamma(\alpha+3)}-\frac{x^{\alpha+3}}{\Gamma(\alpha+4)}-\frac{4 x^{\alpha+4}}{\Gamma(\alpha+5)}$,

Now, we can form the $N$-terms approximation, $N=2$ :

$\Phi_{2}(x)=1+A x+\frac{x^{2}}{2}+\frac{B x^{3}}{6}+\frac{x^{4}}{24}+\frac{C x^{5}}{120}+\frac{x^{\alpha}}{\Gamma(\alpha+1)}+\frac{x^{\alpha+1}}{\Gamma(\alpha+2)}+\frac{x^{\alpha+2}}{\Gamma(\alpha+3)}-\frac{x^{\alpha+3}}{\Gamma(\alpha+4)}-\frac{4 x^{\alpha+4}}{\Gamma(\alpha+5)}$.

Where $A, \mathrm{~B}$ and $C$ can be determined by using boundary condition (39) in $\Phi_{2}(x)$ (see Table 4). 
Table4. Values of $A, B$ and $C$ for different values of $\alpha$ using (49).

\begin{tabular}{ccccc}
\hline & $\alpha=5.25$ & $\alpha=5.50$ & $\alpha=5.75$ & $\alpha=6$ \\
\hline$A$ & 0.9999697551 & 0.9993589934 & 0.9996189375 & 1.000383104 \\
$B$ & 1.002993635 & 1.008525151 & 1.004974548 & 0.9965509942 \\
$C$ & 0.3727393281 & 0.6121554492 & 0.8316703803 & 1.023837383 \\
\hline
\end{tabular}

We compute the absolute error functions $E_{10}(x)=\left|e^{x}-\Phi_{2,5.25}\right|, E_{11}(x)=\left|e^{x}-\Phi_{2,5.50}\right|$ and $E_{12}(x)=\left|e^{x}-\Phi_{2,5.75}\right|$. Where $e^{x}$ is the exact solution of (37) - (39) and $\Phi_{2,5.25}, \Phi_{2,5.50}$ and $\Phi_{2,5.75}$ are approximate solutions of (37) (49) by using (49) at $\alpha=5.25, \alpha=5.50$ and $\alpha=5.75$ respectively. In Fig. 2 we compare the absolute error functions.

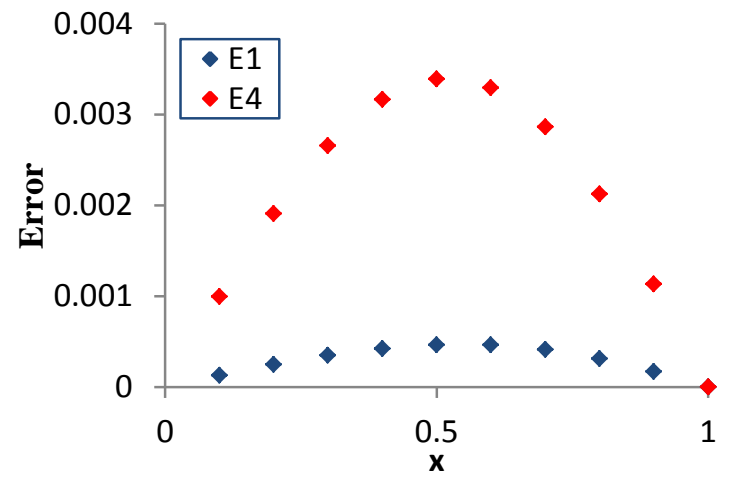

(1a) $\alpha=3.25$

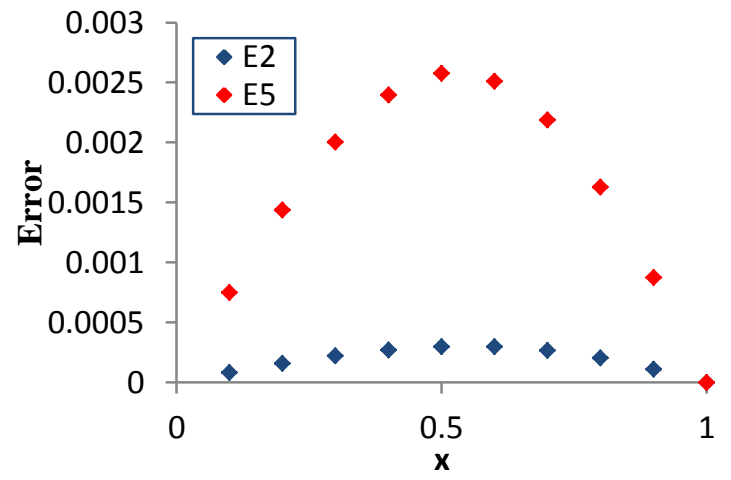

(1b) $\alpha=3.50$

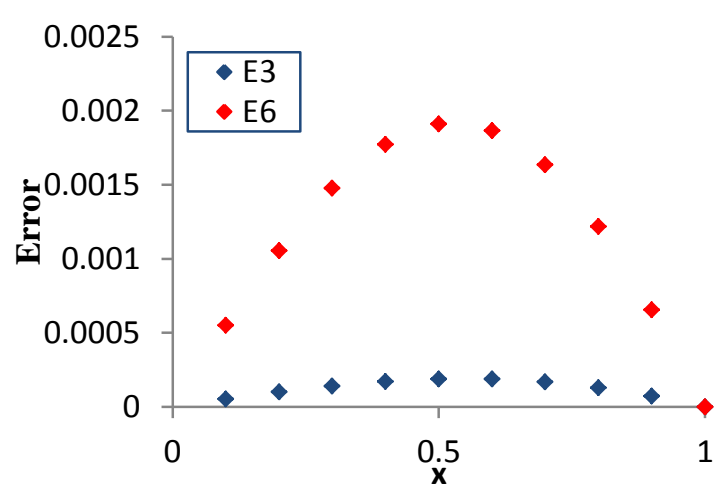

(1c) $\alpha=3.75$

Fig.1. Comparison of absolute error functions $E_{1}(x)-E_{6}(x)$ obtained by ADM and HPM for different $\alpha$.

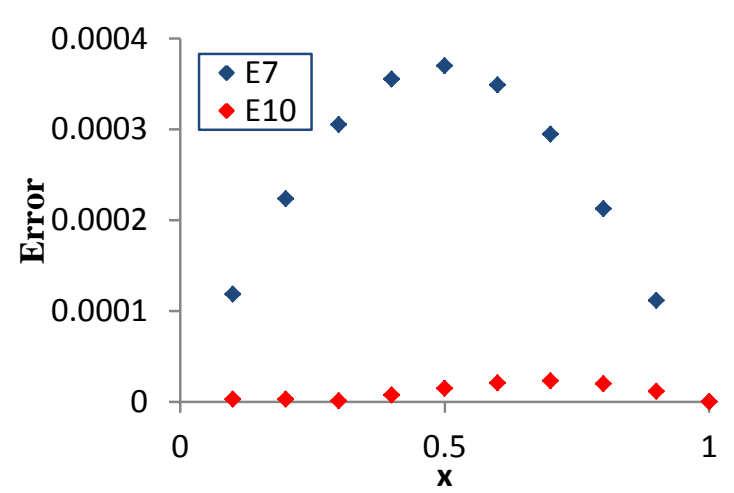

(2a) $\alpha=5.25$

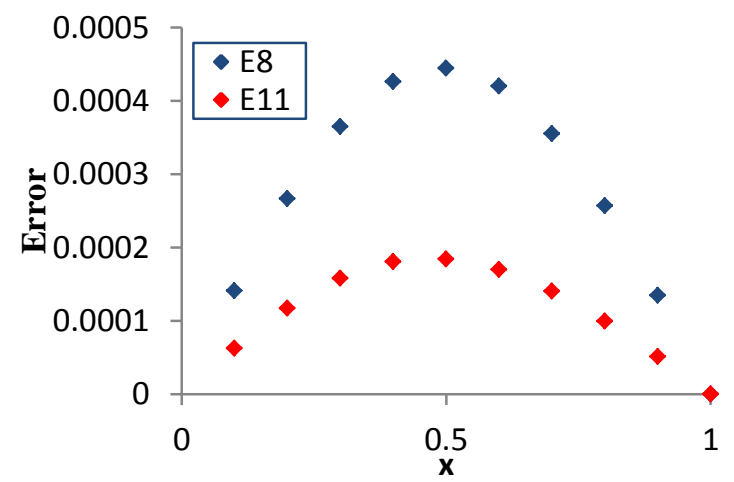

(2b) $\alpha=5.50$ 


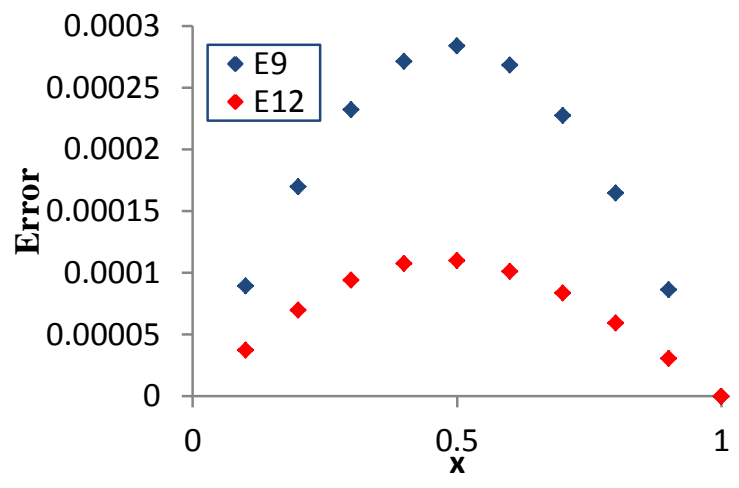

(2c) $\alpha=5.75$

Fig.2. Comparison of absolute error functins $\mathrm{E}_{7}(\mathrm{x})-\mathrm{E}_{12}(\mathrm{x})$ obtained by ADM and HPM for different $\alpha$.

\section{Conclusion}

In this paper, this study showed that the numerical results of most linear fractional integro-differential equations (1) - (3) as follows:

Case (I): If $\gamma=0$ and $\lambda$ is a negative real number, we find ADM is better than HPM (see Fig. 1).

Case (II): If $\gamma=0$ and $\lambda$ is a positive real number, we find HPM is better than ADM (see Fig. 2).

Also it is shown that the accuracy can be improved by more $N$-terms of approximated solutions and by taking more terms in the Taylor expansion of the exponential term.

\section{References}

[1] A. A. Kilbas, H. M. Srivastava, and J. J. Trujillo, Theory and Applications of Fractional Differential Equations, vol. 204 of NorthHolland Mathematics Studies, Elsevier Science B.V., Amsterdam, The Netherlands, 2006.

[2] V. Lakshmikantham, S. Leela, and J. V. Devi, Theory of Fractional Dynamic Systems, Cambridge Scientific, 2009.

[3] K. S. Miller and B. Ross, An Introduction to the Fractional Calculus and Fractional Differential Equations, A Wiley-Interscience Publication, JohnWiley \& Sons, New York, NY, USA, 1993.

[4] I. Podlubny, Fractional Differential Equations, vol. 198 of Mathematics in Science and Engineering, Academic Press, San Diego, Calif, USA, 1999.

[5] K. Diethelm and A.D. Freed, "On the solution of nonlinear fractional order differential equations used in the modeling of viscoelasticity," in Scientific Computing in Chemical Engineering II-Computational Fluid Dynamics, Reaction Engineering and Molecular Properties, F. Keil, W. Mackens, H. Voss, and J. Werther, Eds., pp. 217-224, Springer, Heidelberg, Germany, 1999.

[6] R. Metzler, W. Schick, H.-G. Kilian, and T. F. Nonnenmacher, "Relaxation in filled polymers: a fractional calculus approach," Journal of Chemical Physics, vol. 103, no. 16, pp. 7180-7186, 1995.

[7] L. Gaul, P. Klein, and S. Kemple, "Damping description involving fractional operators," Mechanical Systems and Signal Processing, vol. 5, no. 2, pp. 81-88, 1991.

[8] W. G. Glockle and T. F. Nonnenmacher, "A fractional calculus approach of self-similar protein dynamics," Biophysical Journal, vol. 68 , pp. 46-53, 1995.

[9] R. Hilfert, Applications of Fractional Calculus in Physics, World Scientific, River Edge, NJ, USA, 2000

[10] R. P. Agarwal, M. Benchohra, and S. Hamani, "A survey on existence results for boundary value problems of nonlinear fractional differential equations and inclusions," Acta ApplicandaeMathematicae, vol. 109, no. 3, pp. 973-1033, 2010.

[11] Z. Bai and H. L" u, "Positive solutions for boundary value problem of nonlinear fractional differential equation," Journal of Mathematical Analysis and Applications, vol. 311, no. 2, pp. 495-505, 2005.

[12] Zhenyu Guo, Min Liu, Donglei Wang, Solutions of Nonlinear Fractional Integro-differential Equations with Boundary Conditions, Bulletin of TICMI. 16 (2) ( 2012), 58-65.

[13] Bashir Ahmad, Juan J. Nieto, Existence Results for Nonlinear Boundary Value problems of Fractional Integro-differential Equations with Integral Boundary Conditions, Hindawi Publishing Corporation, Boundary Value problems. (2009) 1-11.

[14] Shaher Momani, Samir Hadid, LYAPUNOV Stability Solutions of Fractional Integro-differential Equations, Hindawi Publishing Corporation, IJMMS. 47 (2004) 2503-2507.

[15] G. Adomian, A review of the decomposition method in applied mathematics, J. Math. Anal. Appl. 135 (1988) 501-544.

[16] G. Adomian, Solving Frontier Problems of Physics: The Decomposition Method, Kluwer Academic Publishers, Boston, 1994

[17] J.H. He, Homotopy perturbation method: a new nonlinear analytic technique, Applied Mathematics and Computation 135 (2003) 73-79.

[18] J.H. He, Homotopy perturbation technique, Computer Methods in Applied Mechanics and Engineering 178 (1999) $257-262$.

[19] J.H. He, A coupling method of homotopy technique and perturbation technique for nonlinear problems, International Journal of Nonlinear Mechanics 35 (1) (2000) 37-43.

[20] Yasir Nawaz, Variational iteration method and homotopy perturbation method for fourth-order fractional integro-differential equations, Computers and Mathematics with Applications, 61 (2011) 2330-2341.

[21] Xindong Zhang, Bo Tang, Yinnian He, Homotopy analysis method for higher-order fractional integro-differential equations, Computers and Mathematics with Applications, 62 (2011) 3194-3203.

[22] Aytac Arikoglu, Ibrahim Ozkol, Solution of fractional integro-differential equations by using fractional differential transform method, Chaos, Solitons and Fractals, 40 (2009) 521-529. 
[23] Shaher Momani, Muhammad Aslam Noor, Numerical methods for fourth-order fractional integro-differential equations, Applied Mathematics and Computation, 182 (2006) 754-760.

[24] V. Seng, K. Abbaoui, Y. Cherruault, Adomian's polynomials for nonlinear operators, Math. Comput. Modell. 24 (1) (1996) 59-65.

[25] Y. Cherruault, Convergence of Adomian's method, Kybernetes 18 (1989) 31-38.

[26] A. Re'paci, Nonlinear dynamical systems: on the accuracy of Adomian's decomposition method, Appl. Mth. Lett. 3 (3) (1990) 3539.

[27] Y. Cherruault, G. Adomian, Decomposition methods: a new proof of convergence, Math. Comput. Modell. 18 (1993) 103-106.

[28] K. Abbaoui, Y. Cherruault, New ideas for proving convergence of decomposition methods, Comput. Math. Appl. 29 (7) (1996) $103-108$.

[29] K. Abbaoui, Y. Cherruault, Convergence of Adomian's method applied to differential equations, Comput. Math. Appl. 28 (5) (1996) 103-109.

[30] J.H. He, Non-Perturbative Methods for Strongly Nonlinear Problems, Dissertation, de-Verlag im Internet GmbH, Berlin, 2006. 